\title{
Magnetism and Specific Heat of TmRhX (X = Ga, Ge) Compounds
}

\author{
S. BARAN ${ }^{a}$, J. PRZEWOŹNIK ${ }^{b}$ AND A. SzYTUŁA ${ }^{a, *}$ \\ ${ }^{a}$ M. Smoluchowski Institute of Physics, Jagiellonian University, W.S. Reymonta 4, 30-059 Kraków, Poland \\ ${ }^{b}$ AGH University of Science and Technology, Faculty of Physics and Applied Computer Science \\ Department of Solid State Physics, al. A. Mickiewicza 30, 30-059 Krakow, Poland
}

\begin{abstract}
Magnetic and specific heat data of the TmRhX $(\mathrm{X}=\mathrm{Ga}, \mathrm{Ge})$ compounds are reported. These compounds crystallize in an orthorhombic crystal structure of the TiNiSi-type (space group Pnma). Magnetic data indicate that the compounds are antiferromagnets with the Néel temperature $T_{\mathrm{N}}$ equal to $3.9 \mathrm{~K}$ for TmRhGa and $6.0 \mathrm{~K}$ for TmRhGe. Magnetic susceptibility of TmRhGe has an additional peak at $T_{\mathrm{t}}=10.6 \mathrm{~K}$. In TmRhGe temperature dependence of $\chi^{\prime \prime}(T)$, the positions of both the peaks at $T_{\mathrm{N}}$ and $T_{\mathrm{t}}$ change with frequency indicating a relaxation process. These data suggest that with the decreasing temperature, first a cluster glass state and next the long-range aniferromagnetic order exists.
\end{abstract}

DOI: 10.12693/APhysPolA.124.998

PACS: 75.30.-m, 75.30.kz, 75.40.Cx, 75.47.Np

\section{Introduction}

This paper is a part of systematic studies of magnetic properties of ternary compounds containing the rare-earth $(\mathrm{R})$ elements, transition metals $(\mathrm{T})$ and $p$-electron elements $(\mathrm{X})$. In the $\mathrm{R}-\mathrm{T}-\mathrm{X}$ systems several hundreds of ternary compounds having different composition are observed [1]. In the last years investigations concentrate on phases with 1:1:1 stoichiometry. The magnetic properties of RTX compounds with $\mathrm{R}=\mathrm{Gd}-\mathrm{Er}$ are well known while there is still some lack of knowledge about those containing $\operatorname{Tm}[2,3]$.

The TmRhX (X = Ga, Ge) compounds investigated in this work crystallize in the orthorhombic TiNiSi-type crystal structure $[4,5]$. Our previous magnetic and neutron diffraction data have shown that both the compounds are antiferromagnets below $T_{\mathrm{N}}$ equal to $3.9 \mathrm{~K}$ and $6.2 \mathrm{~K}$ for TmRhGa and TmRhGe, respectively [6, 7]. In purpose to understand more deeply magnetic properties of these compounds the AC and DC magnetic as well as specific heat measurements have been performed.

\section{Experimental and results}

Both the polycrystalline samples were synthesized by arc melting under argon atmosphere. High purity component elements were used in the stoichiometric ratio 1:1:1. In order to obtain a homogeneous material, the samples were annealed at $800^{\circ} \mathrm{C}$ for 1 week.

The samples were examined by X-ray powder diffraction performed at room temperature on a Philips PW-3710 X'PERT diffractometer using $\mathrm{Cu} K_{\alpha}$ radiation.

The DC magnetic measurements were carried out using a vibrating sample magnetometer (VSM) option of the

*corresponding author; e-mail: andrzej.szytula@uj.edu.pl
Quantum Design PPMS platform. Magnetic susceptibility was measured over temperature interval $1.9-350 \mathrm{~K}$ in order to determine phase transition temperatures, effective magnetic moment $\mu_{\text {eff }}$ and paramagnetic Curie temperature $\theta_{\mathrm{p}}$. Magnetization curves were measured up to $90 \mathrm{kOe}(9 \mathrm{~T})$ at $1.9 \mathrm{~K}$ in purpose to determine magnetic moment in the ordered state.

The AC magnetic susceptibility $\chi_{\mathrm{AC}}=\chi^{\prime}-\mathrm{i} \chi^{\prime \prime}$ (where $\chi^{\prime}$ is the real and $\chi^{\prime \prime}$ the imaginary component) was measured at several chosen frequencies between $50 \mathrm{~Hz}$ and $5 \mathrm{kHz}$ in the temperature range $1.9-20 \mathrm{~K}$ with the use of an AC magnetic susceptibility (ACMS) option of the Quantum Design PPMS platform.

The heat capacity measurements were carried out by a two-tau relaxation method in the temperature range 1.9$17 \mathrm{~K}$ using a heat capacity option (HC) of the Quantum Design PPMS platform.

The analysis of X-ray data obtained at room temperature indicates that the investigated compounds have an orthorhombic crystal structure of the NiTiSi-type with the lattice parameters being in good agreement with previously reported values $[4,5]$.

The temperature dependences of DC magnetic susceptibilities measured at $H=1 \mathrm{kOe}$ (see Fig. 1) obey the Curie-Weiss law with positive values of paramagnetic Curie temperatures and effective magnetic moments being close to the free $\mathrm{Tm}^{3+}$ ion value $\left(7.56 \mu_{\mathrm{B}}\right)$. At low temperatures a maximum at $3.9 \mathrm{~K}$ is observed in TmRhGa. For TmRhGe both the zero field cooling (ZFC) and field cooling (FC) curves have a maximum at $6 \mathrm{~K}$ typical of phase transition from an antiferro- to a paramagnetic state. This result is in a good agreement with the previously reported data $(6.64 \mathrm{~K})$ [7]. Below $10.6 \mathrm{~K}$ a difference between $\mathrm{ZFC}$ and $\mathrm{FC}$ curves is observed.

The magnetization vs. magnetic field curves (see lower insets in Fig. 1) have a metamagnetic character with criti- 


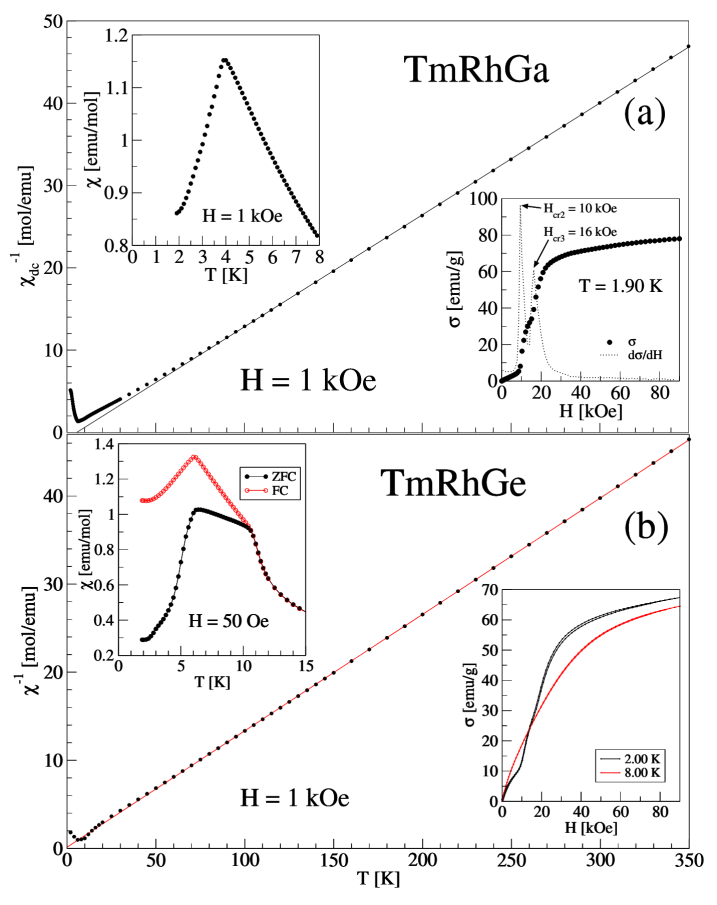

Fig. 1. Temperature dependence of reciprocal magnetic susceptibility measured at $1 \mathrm{kOe}$ applied magnetic field for TmRhGa (upper part) and TmRhGe (lower part). Insets show: the upper ones - temperature dependence of magnetic susceptibility at low temperatures; the lower ones - magnetization curves up to $90 \mathrm{kOe}$ at low temperatures.
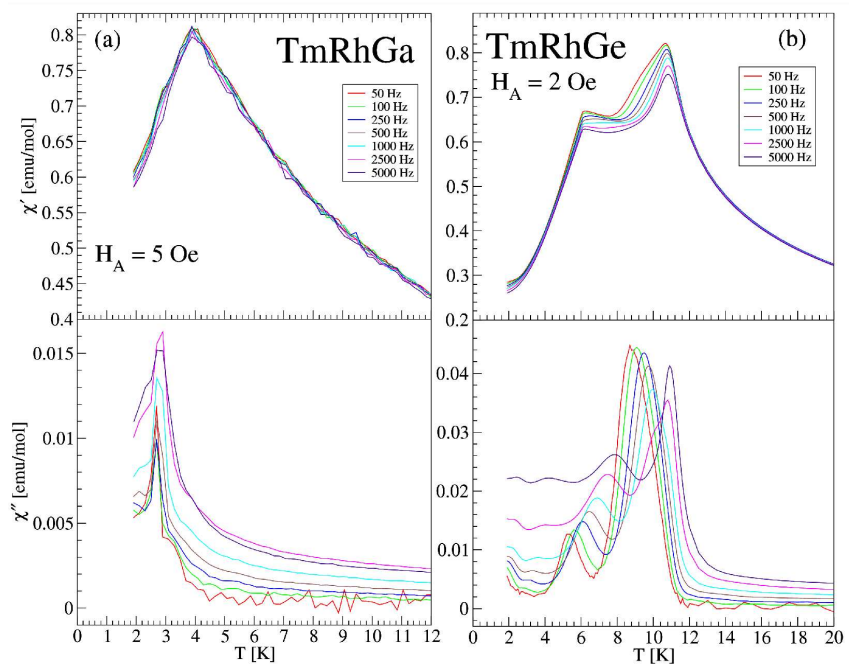

Fig. 2. Temperature dependence of the real $\chi^{\prime}$ (upper part) and imaginary $\chi^{\prime \prime}$ (lower part) component of AC magnetic susceptibility in TmRhGa (left part) and TmRhGe (right part).

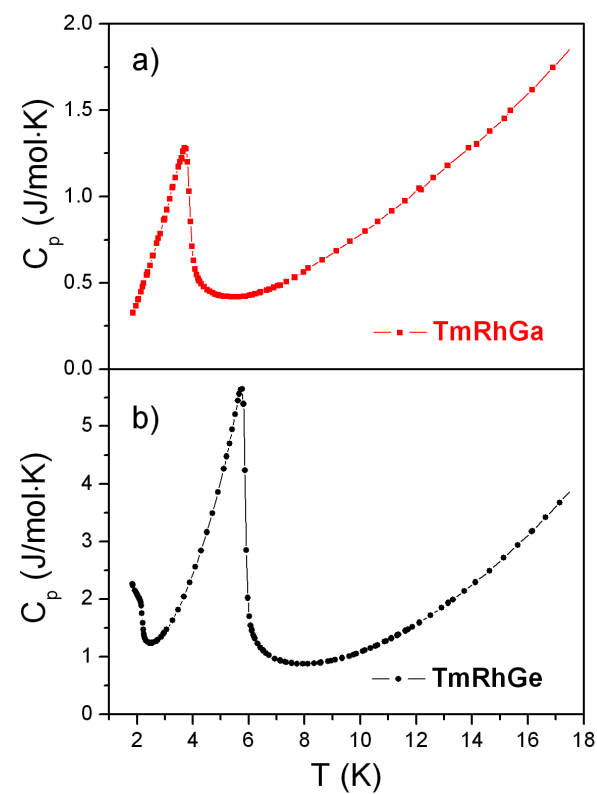

Fig. 3. Specific heat temperature dependence in TmRhGa and TmRhGe.

cal fields $H_{\text {cr }}$ equal to $0.08(3), 10.0(5)$ and $16.0(5) \mathrm{kOe}$ for TmRhGa and 0.8(2), 11(1) and 19(1) kOe for TmRhGe. The magnetization curves do not saturate at magnetic field of $90 \mathrm{kOe}$ and $T=1.9 \mathrm{~K}$. The values of Tm magnetic moments at $T=1.9 \mathrm{~K}$ and $H=90 \mathrm{kOe}$ are clearly smaller than the free $\mathrm{Tm}^{3+}$ ion value $\left(7.0 \mu_{\mathrm{B}}\right)$ (see Table).

Temperature dependences of the real $\chi^{\prime}$ and imaginary $\chi^{\prime \prime}$ component of AC magnetic susceptibility for both the investigated compounds are shown in Fig. 2.

In TmRhGa the real component $\chi^{\prime}$ has a frequency independent maximum at $3.9 \mathrm{~K}$ while the temperature of maximum of imaginary component slightly increases from $2.7 \mathrm{~K}$ to $2.9 \mathrm{~K}$ with increasing frequency. In case of TmRhGe two maxima in $\chi^{\prime}(T)$ and $\chi^{\prime \prime}(T)$ temperature dependence are observed. In $\chi^{\prime}(T)$ these almost temperature independent maxima appear at $T_{1}=6.2(1) \mathrm{K}$, while the second, at $T_{2}$, increases from 10.64 to $10.84 \mathrm{~K}$ when frequency increases from $50 \mathrm{~Hz}$ to $5 \mathrm{kHz}$, respectively. This dependence is described by a semi-empirical dimensionless parameter

$$
K=\frac{\Delta T}{[T \Delta(\log f)]} .
$$

The value $K$ equal to 0.0094 is typical for metallic spin-glass system [8]. In contrary, the maxima in $\chi^{\prime \prime}(T)$ depend strongly on frequency in TmRhGe. The temperature $T_{1}$ of the first maximum increases from 5.3(1) $\mathrm{K}$ for $f=50 \mathrm{~Hz}$ to $7.9(1) \mathrm{K}$ for $f=5 \mathrm{kHz}$ while the temperature $T_{2}$ of the second maximum shifts from 8.7(1) K for $f=50 \mathrm{~Hz}$ to $10.9(1) \mathrm{K}$ for $f=5 \mathrm{kHz}$. The frequency dependence of $T_{1}$ and $T_{2}$ points to the slow relaxation of the spin system and suggests strong magnetocrystalline anisotropy. The relation of $\frac{1}{f}$ versus $\frac{1}{T_{i}}$ has been analyzed by the Arrhenius equation 


$$
\tau=\tau_{0} \exp \left(-\frac{E_{\mathrm{a}}}{k_{\mathrm{B}} T}\right),
$$

where $\tau_{0}$ is a characteristic microscopic time and $E_{\text {a }}$ is an activation energy, yielding the following values: $\tau_{0}=10^{-8} \mathrm{~s}$ and $E_{\mathrm{a}}=103.5 \mathrm{~K}(6.45 \mathrm{meV})$ for $T_{1}$ and $\tau_{0}=10^{-12} \mathrm{~s}$ and $E_{\mathrm{a}}=287.0 \mathrm{~K}(17.9 \mathrm{meV})$ for $T_{2}$.

Figure 3 shows the specific heat versus temperature plots for both compounds at low temperatures. The prominent $\lambda$-like peaks at $3.7 \mathrm{~K}$ for TmRhGa and $5.75 \mathrm{~K}$ for TmRhGe, indicative of a long-range magnetic order, are present. In TmRhGe additional anomaly at $2.2 \mathrm{~K}$ is observed, while no extra anomalies were found for TmRhGa below $T_{\mathrm{N}}$. The specific heat measurement on TmRhGe did not show any observable anomaly around 10.6 K, like it was the case for TbAuIn [9] showing also cluster-glass state above the long-range ordering transition.

TABLE

Magnetic properties of $\operatorname{TmRhX}(\mathrm{X}=\mathrm{Ga}, \mathrm{Ge})$ : the Néel temperature $\left(T_{\mathrm{N}}\right)$, the transition temperature $\left(T_{\mathrm{t}}\right)$, the paramagnetic Curie temperature $\left(\theta_{\mathrm{p}}\right)$, the effective magnetic moment $\left(\mu_{\mathrm{eff}}\right)$, the magnetic moment $(\mu)$ at $H=90 \mathrm{kOe}$ and $T=1.9 \mathrm{~K}$, and the critical magnetic field $\left(H_{\mathrm{cr}}\right)$. Theoretical value for a free $\mathrm{Tm}^{3+}$ ion is $7.56 \mu_{\mathrm{B}}\left(\mu_{\text {eff }}\right)$ and $7.0 \mu_{\mathrm{B}}(\mu)$. Experimental methods: $\mathrm{DC}, \mathrm{AC}$ - magnetic and hc - heat capacity measurements.

\begin{tabular}{c|c|c|c|c|c|c|c}
\hline \hline Compound & Method & $T_{\mathrm{N}}[\mathrm{K}]$ & $T_{\mathrm{t}}[\mathrm{K}]$ & $\theta_{\mathrm{p}}[\mathrm{K}]$ & $\mu_{\mathrm{eff}}\left[\mu_{\mathrm{B}}\right]$ & $\mu\left[\mu_{\mathrm{B}}\right]$ & $H_{\mathrm{cr}}[\mathrm{kOe}]$ \\
\hline TmRhGa & $\mathrm{dc}$ & 3.9 & & 5.6 & 7.67 & 5.0 & $0.08,10.16$ \\
& $\mathrm{AC}$ & 3.9 & 2.7 & & & & \\
& $\mathrm{hc}$ & 3.7 & & & & & \\
\multirow{5}{*}{ TmRhGe } & $\mathrm{DC}$ & 6.0 & 10.64 & 1.0 & 7.81 & 4.20 & $0.8,11,19$ \\
& $\mathrm{AC}$ & 6.0 & 10.64 & & & & \\
& $\mathrm{hc}$ & 5.75 & 2.2 & & & &
\end{tabular}

\section{Discussion}

The results presented in this work, in particular the $\mathrm{AC}$ magnetic data, indicate complex magnetic properties in investigated TmTX compounds.

In TmRhGa all the data (AC- and DC-magnetic as well as specific heat) indicate existence of a long-range magnetic order below $3.9 \mathrm{~K}$. The long-range magnetic order was also detected by neutron diffraction [6]. Temperature dependence of $\chi^{\prime \prime}$ gives additional anomaly at $2.7 \mathrm{~K}$. The lack of neutron diffraction between $1.5 \mathrm{~K}$ and paramagnetic state [7] prevents from determining the nature of the latter anomaly.

In TmRhGe the $\chi_{\mathrm{DC}}(\mathrm{FC})$ temperature dependence has only one distinct anomaly at $6 \mathrm{~K}$ while ZFC data show two anomalies: at 6 and $10.64 \mathrm{~K}$ (see Fig. 1) the neutron diffraction data indicate existence of magnetic order below $6 \mathrm{~K} \mathrm{[7].} \mathrm{These} \mathrm{data} \mathrm{are} \mathrm{similar} \mathrm{to} \mathrm{those} \mathrm{ob-}$ served in TbAuIn [9] and in the isostructural RIrSi $(\mathrm{R}=$ Tb, Dy, Ho) compounds [10]. The difference between ZFC and FC curves above $T_{\mathrm{N}}$ is due to a short-range magnetic order. Two anomalies in $\chi^{\prime}(T)$ and $\chi^{\prime \prime}(T)$ curves are observed at 6.0 and $10.6 \mathrm{~K}$. These temperatures have been found to be frequency dependent. Such a behavior is typical for relaxation process. The Arrhenius equation applied to the relation between frequency and temperatures for both maxima provides two sets of $\tau_{0}$ and $E_{\mathrm{a}}$ parameters, where activation energy for the transition at $T_{2}$ is three times larger than that for the transition at $T_{1}$. Thus, one may conclude from the $\mathrm{AC}$ and DC measurements that with decreasing temperature a metallic spin glass cluster formation process begins at $T_{2}\left(=T_{\mathrm{t}}\right)$ followed by a long-range magnetic order appearing at $T_{1}\left(=T_{\mathrm{N}}\right)$.

Positive values of the paramagnetic Curie temperature observed in both compounds are typical for the layered structures in which the strong ferro-coupling is in plane and antiferro- between the planes. Investigated compounds crystallize in orthorhombic TiNiSi-type structure. The structure can be displayed as an array of layers (at $y=1 / 4$ and $3 / 4$ ) piled up along the $b$-axis direction. Such structure may cause magnetocrystalline anisotropy, which in turn influences relaxation properties.

The neutron diffraction data indicate that in both the compounds the Tm magnetic moments - close to the free $\mathrm{Tm}^{3+}$ ion value $\left(7.0 \mu_{\mathrm{B}}\right)$ - form a sine-wave modulated structure at zero applied magnetic field. The structure is described by the propagation vector $\boldsymbol{k}=\left(0, k_{y}, 0\right)$ with $k_{y}$ equal to $0.4222(3)$ in TmRhGa [6] and 0.5 in TmRhGe [7].

In contrary, the values of Tm moments determined from magnetization data at $H=90 \mathrm{kOe}$ are smaller than those found from diffraction measurements indicating complex magnetic structures after metamagnetic transition.

The smaller value of the Néel temperature for TmRhGa than for TmRhGe means that magnetic interactions are influenced by interatomic distances.

In summary, this work reports magnetic macroscopic properties and heat capacity of $\operatorname{TmRhX}(\mathrm{X}=\mathrm{Ga}$, $\mathrm{Ge})$. Especially interesting results have been found for 
TmRhGe where the difference between ZFC and FC DC magnetic susceptibility curves suggests strong domain wall pinning. Taking into account also the $\mathrm{AC}$ magnetic data reveals complex magnetic properties: with decrease of temperature cluster spin glass formation process is observed followed by a transition to long-range magnetically ordered state at lower temperatures.

\section{Acknowledgments}

This work is supported by the Ministry of Science and Higher Education in Poland under grant no. N N202 2010 39. The research was carried out with the equipment purchased thanks to the financial support of the European Regional Development Fund in the framework of the Polish Innovation Economy Operational Program (contract no. POIG.02.01.00-12-023/08).

\section{References}

[1] E. Parthé, B. Chabot, in: Handbook on the Physics and Chemistry of Rare Earths, Eds. K.A. Gschneidner Jr., L. Eyring, Elsevier Sci., Amsterdam 1984, Vol. 6, Ch. 48, p. 113.
[2] A. Szytuła, Crystal Structures and Magnetic Properties of RTX Rare Earth Intermetallics, Wydawnictwo Uniwersytetu Jagiellońskiego, Kraków 1998.

[3] A. Szytuła, J. Leciejewicz, Handbook of Crystal Structures and Magnetic Properties of Rare Earth Intermetallics, CRC Press, Boca Raton 1994.

[4] F. Hulliger, J. Alloys Comp. 239, 131 (1996).

[5] A.V. Morozkin, Yu.D. Seropegin, I.A. Sviridov, J. Alloys Comp. 270, L4 (1998).

[6] S. Baran, A. Hoser, A. Szytuła, J. Magn. Magn. Mater. 335, 97 (2013).

[7] W. Bażela, M. Hofmann, S. Baran, B. Penc, A. Szytuła, A. Zygmunt, Acta Phys. Pol. A 97, 819 (2000).

[8] J.A. Mydosh, Spin Glass: An Experimental Introduction, Taylor and Francis, London 1993.

[9] Ł. Gondek, A. Szytuła, M. Bałanda, W. Warkocki, A. Szewczyk, M. Gutowska, Solid State Commun. 136, 26 (2005).

[10] R. Duraj, A. Szytuła, Acta Phys. Pol. A 123, 773 (2013). 